\title{
Realidades y percepciones culturales sobre cambio climático en las comunidades étnicas del Chocó Biogeográfico
}

\section{Realities and cultural perceptions of climate change in ethnic communities in the Chocó Biogeográfico}

\author{
William Klinger Brahan*
}

\section{Resumen}

Las continuas emisiones de gases originados en las actividades productivas del hombre y sus consecuentes efectos sobre las condiciones de clima en el mundo, hoy son reconocidas por la población de manera generalizada y después de muchos años de ser vistas como especulaciones, se ven ahora como una amenaza contra la estabilidad ambiental del planeta, contra la seguridad de la población y contra la soberanía alimentaria de las comunidades. Con este documento se pretende mostrar las realidades y las percepciones del fenómeno de cambio climático en las comunidades étnicas del Chocó Biogeográfico, amparado en las observaciones directas de su comportamiento, así como en los resultados de diálogos y encuestas aplicadas a líderes de la región. El conocimiento tradicional, pilar fundamental de la identidad cultural de negros e indigenas del Pacífico, cobra fuerza y vigor en la percepción comunitaria del fenómeno y aspectos como la religiosidad, los mitos y las leyendas, los sistemas productivos tradicionales y la relación del hombre con la naturaleza aparecen no sólo como elementos que explican el cambio climático, sino también sus efectos. De igual manera, se percibe al interior de las comunidades que el uso de la naturaleza conforme a las reglas de la cosmovisión indígena y negra, que aplique las normas sociales y los reglamentos comunitarios, son además de una forma de defender su presencia en éste, maneras efectivas de mitigar los efectos del cambio climático, por lo que el fortalecimiento de las organizaciones étnico territoriales se ve como una necesidad para enfrentar el problema.

Palabras clave: Adaptación, Cambio climático, Comunidades étnicas, Percepciones culturales, Procesos comunitarios.

\begin{abstract}
Continuous emissions originate gases in productive activities of man and his consequent effects on weather conditions in the world today are recognized by the population generally, and after many years of being seen as speculation, are now as a threat to environmental stability of the planet, the security of the population and against food sovereignty of communities. With this document I intend to show the realities and perceptions of the phenomenon of climate change in the ethnic communities of Chocó Biogeográfico, protected by direct observations of their behavior, as well as the results of dialogues and surveys of male and female leaders in the region. Traditional knowledge, a fundamental pillar of the cultural identity of black and indigenous Pacific, gaining strength and vigor in the community perception of the phenomenon and issues such as religion, myths and legends, traditional production
\end{abstract}

* Ingeniero Forestal, Profesor Titular de la Universidad Distrital Francisco José de Caldas, Director General del Instituto de Investigaciones Ambientales del Pacífico. e-mail: wklinger@iiiap.org.co

Recibido:9 de diciembre de 2013 Aprobado: 19 de febrero de 2014 


\section{Cambio climático y percepciones culturales. W Klinger}

systems and man's relationship with nature appear as not only factors behind climate change but also its effects. Similarly, it is perceived within communities that use of nature under the rules of the indigenous and black worldview, to implement social norms and social regulations, are also a way to defend their presence in it, effective ways to mitigate the effects of climate change, so the strengthening of ethnic organizations is seen as a need to address the problem.

Keywords: Adaptation, Climate change, Community processes, Cultural perceptions, Ethnic communities.

\section{Factores que han promovido la toma de conciencia de la comunidad frente al cambio climático}

La vivencia de evidentes fenómenos asociados con las condiciones climáticas de la región y la ocurrencia de fenómenos relacionados con el clima, ha despertado notoriamente la conciencia de las poblaciones asentadas en el Chocó Biogeográfico frente al cambio climático y ha favorecido el papel de los educadores ambientales hacia el reconocimiento comunitario de la situación. Sin embargo, como es de esperarse, también son evidentes las prácticas productivas cada vez más lejanas a las consideraciones culturales de la población, que muestran una inequívoca ruptura con la oferta ambiental del territorio; la gente en la búsqueda de explicaciones a la ocurrencia de desastres naturales, no puede desligar los efectos del cambio climático de las conductas ambientales de la ciudadanía en general, por lo que no puede explicarse los fenómenos en relación exclusiva con estos cambios.

Fenómenos como la masiva mortandad de peces de variadas especies que registró en el año 2009 el municipio del Alto Baudó en el departamento del Chocó; en ese mismo año la creciente del río San Juan por encima de los estándares históricos, situación que condujo a una de las mayores crisis alimentarias de la subregión y a la desaparición casi completa de asentamientos ribereños; la avalancha de 1999, que por represamiento del río Cupica arrasó con esta población del municipio de Bahía Solano, empujada mar afuera; los graves daños a las viviendas y la infraestructura del municipio de Olaya Herrera ocasionados por la corriente de los ríos Patía y Sanquianga; los altos picos de calor soportados por los habitantes de Quibdó durante algunos períodos en los años 2008 y 2009; las permanentes inundaciones en el año registradas en poblaciones como Riosucio, en donde sus habitantes vivieron literalmente sobre canoas gran parte del tiempo, entre muchos otros fenómenos, hoy son claramente asociados por las comunidades a situaciones de cambio climático. Se aprecia aquí una percepción del cambio climático que lo relaciona con desastres naturales y con la variación de los parámetros climáticos de precipitación y temperatura; un alto porcentaje de nuestros líderes comunitarios así lo entiende.

De la misma manera, el IIAP ha investigado otros eventos menos evidentes para la comunidad en relación directa con el cambio climático, pero igualmente importantes desde el punto de vista de conservación de la biodiversidad, por el Instituto de Investigaciones Ambientales del Pacífico (IIAP) y puesto en conocimiento de las comunidades con interpretaciones por parte de ellas, que de alguna manera involucran aspectos relativos con el fenómeno, la reducción de la superficie de algunos ecosistemas estratégicos de la región, el desplazamiento de algunas especies de aves migratorias, que registran una gran movilidad desde ecosistemas cercanos a la línea costera hasta otros ecosistemas ubicados varios kilómetros aguas arriba del río Atrato, la notoria escasez de algunas especies de amplia cultura de uso local en municipios de alta vocación forestal, el reporte de un gran número de accidentes ofídicos por un notable aumento de serpientes venenosas, la aparición de plagas en cultivos altamente productivos y de enorme importancia local como el chontaduro, son entre otros, eventos que llaman la atención desde el punto de vista académico y están estrechamente conectados con las condiciones de bienestar colectivo de las comunidades. En esta percepción impera la relación del cambio climático con el deterioro ambiental, la escasez de recursos naturales, la reducción de alimentos y la aparición de plagas.

Otro porcentaje de nuestros líderes perciben el cambio climático en relación con la insalubridad y aparición de epidemias en general y con el calendario general de siembras y cosechas. A pesar de estos altos niveles de conciencia ciudadana frente al cambio climático, no faltan quienes dentro de la comunidad perciban todas las manifestaciones de la mezcla cambio climático y factores antropogénicos con una situación normal de la naturaleza que va y viene presentando ciclos y periodicidades que no les generan ningún tipo de alarma.

Estos fenómenos y eventos reciben eso sí, muy diversas interpretaciones y explicaciones por parte de la población, las cuales se amparan en el conocimiento de la naturaleza y la cosmovisión de las comunidades, que influyen a su vez en la forma de uso de los recursos naturales. Las diferencias de interpretación se acentúan sobre todo en función del tipo de grupo étnico y del tipo de poblamiento que tenga el territorio; por una parte las marcadas diferencias de cosmovisión entre los negros, los indígenas y las comunidades campesinas que están mediadas por el nivel de conocimiento tanto tradicional como académico de unas y otras, y de igual manera, aporta a la diferencia de apreciaciones culturales de los fenómenos asociados con el cambio climático, si se trata de población rural o urbana, y si la población está 


\section{Bioetnia Volumen 11, 2014}

asentada en ecosistemas marino costeros o continentales, pues es evidente que no sólo los efectos son distintos, sino también el grado de vulnerabilidad de cada una de estas poblaciones y sus posibilidades de enfrentar con éxito la ocurrencia de un fenómeno natural de grandes dimensiones.

Las interpretaciones de los fenómenos climáticos y sus efectos sobre la población, están mediados por la cosmovisión y el conocimiento y se mueven desde aspectos mágicos y religiosos hasta aspectos ampliamente reconocidos por la comunidad académica contemporánea, pero lo más importante es que el cambio climático se reconoce, se relaciona su existencia con malas prácticas productivas y se visualiza como un fenómeno de efectos globales dentro de nuestras comunidades. Con ocasión de esta invitación, se adelantaron conversaciones y se aplicaron 30 encuestas a líderes de la región, encontrando que 100\% reconoce el fenómeno de cambio climático y atribuye a él, en parte, sequías prolongadas, inundaciones permanentes, lluvias y soles intensos, desbordamiento de ríos y quebradas, pérdida de cosechas y reducción generalizada de la oferta de recursos naturales.

Las comunidades étnicas asocian los desastres naturales, el ataque de plagas y la escasez de recursos naturales con el cambio climático, y se lo explican de diversas maneras; algunos miembros de comunidades indígenas atribuyen este tipo de situaciones a molestias de los dioses por el desarrollo de prácticas productivas no respetuosas de su cosmovisión; así explican por ejemplo, la aparición de plagas del chontaduro. Miembros de comunidades negras ubican como razones de estas mismas situaciones el cambio de uso del territorio y la intensidad de los aprovechamientos, así explica la desecación de humedales y quebradas por ejemplo.

\section{Afectación del modo de vida de las comunidades por efectos del cambio climático}

Los fenómenos asociados con el cambio climático, sin duda han generado afectaciones significativas en el modo de vida de los pobladores del Chocó Biogeográfico, las cuales van desde extremos problemas de desarraigo y desplazamiento territorial con los consecuentes inconvenientes que acompañan este tipo de situaciones, hasta el cambio súbito de actividades productivas orientadas a la generación de ingresos básicos para la sobrevivencia de poblaciones y familias enteras, pasando por asuntos relativos a tener cada vez mayores esfuerzos físicos para lograr el mismo rendimiento en el aprovechamiento de los recursos naturales disponibles en su territorio.

La ocupación del territorio promueve la realización de actividades productivas alrededor de las viviendas de los pobladores, con distancias que están finamente calculadas por ellos, con base en su capacidad de esfuerzo físico para hacer uso de los recursos existentes, situaciones de desastres naturales como el desbordamiento del río Mira en febrero del 2008, que generó afectación de grandes extensiones de tierra, que le fueron usurpadas por la naturaleza a millares de personas que además quedaron sin techo, por solo citar un ejemplo, produjo cambios significativos en el modo de vida de estos habitantes del Pacífico, modo de vida con el cual se asocia una cultura del agua que exige tener bajo su manejo un territorio con características especiales.

Para casos como este que son muchos en la región, mientras se producen condiciones adecuadas para el retorno a sus tierras, en caso de ser posible, se ven pobladores históricamente rurales, alojados en los centros urbanos, sin ninguna actividad productiva que desarrollar, situación que conduce al deterioro de las condiciones de vida de los familiares y amigos que solidariamente apoyan a quienes sufrieron calamidades, alojándolos en sus casas en situación de temporalidad. Muchos otros se quedan en los centros urbanos, engrosando los cordones de miseria de las ciudades grandes e intermedias, caracterizados por la mendicidad y la delincuencia. Buena parte de los afectados por la avalancha de lodo del río Mira permanecen en Tumaco en espera de que se den las condiciones necesarias para su retorno.

Las comunidades que tienen mejor suerte retornan a las tierras tan pronto cesan los embates de la naturaleza, poniendo en riesgo sus vidas ante la inminente amenaza de repetición del fenómeno, pero es claro, que es lo único posible para habitar una tierra propia, la cual se conoce y de la cual se vive. Al llegar a los sitios que históricamente han habitado, las tierras dedicadas a la producción en cultivos de pan coger ya no se encuentran; los ríos, quebradas y ciénagas donde pescaban se encuentran secos y/o sedimentados y en consecuencia, en la mayoría de los casos, las comunidades étnicas deben cambiar abruptamente de actividad productiva principal que desarrollan, lo que los lleva a un proceso de adaptación que requiere tiempo y genera pérdida de dinero por bajo rendimiento.

Otra forma de cambio de vida en las comunidades étnicas del Chocó Biogeográfico está representada en la cada vez menor disponibilidad de tiempo para el descanso; la escasez de los recursos conduce a la inversión de mayores cantidades de tiempo para lograr jornadas de aprovechamiento exitosas, es decir, similares a las de antes. Según los testimonios de pescadores en Pizarro y Bahía Solano, hoy se debe invertir el doble del tiempo para alcanzar los mismos volúmenes de producción de antes en las faenas de pesca; se debe ir mucho más lejos o permanecer más horas en el mar, situación que atribuyen a los cambios de temperatura del mar y a la invasión que hacen los barcos pesqueros a las zonas dedicadas a la pesca artesanal, tareas relacionadas con el control de la pesca marítima ilegal y el aumento de las millas para zonas exclusivas de pesca 


\section{Cambio climático y percepciones culturales. W Klinger}

artesanal en los municipios del Chocó Biogeográfico, se avizoran como de gran importancia para lograr una mejor adaptación de las comunidades a los efectos que sobre ellas produce el cambio climático.

Las comunidades étnicas ubican también como cambios en sus modos de vida, el hecho de llevarla en condiciones cada vez más evidentes de marginalidad y pobreza, lo que trae consigo problemas de insalubridad, bajos niveles de educación y falta de disfrute de escenarios naturales, así como baja calidad del tiempo de esparcimiento; estos elementos fueron claramente identificados por unos pocos encuestados, pero parece claro que constituye en esencia, cambios dramáticos en las condiciones de vida iniciales de la población. Denuncian que asociadas con las prácticas productivas invasoras como muchos las llaman, trae consigo problemas de deterioro del tejido social, crecimiento irregular de las familias, más dificultad de acceso a los servicios públicos y de salud, prostitución y pérdida del territorio, por físico abandono.

\section{Los procesos comunitarios de adaptación al cambio climático como respuestas individuales vitales e inteligentes, pero no planificadas}

Las respuestas individuales de los miembros de grupos étnicos negros e indígenas al cambio climático dependen de las oportunidades que el Estado, los familiares y/ los amigos les puedan ofrecer para habitar otros espacios geográficos, en su preparación para desarrollar otras actividades productivas, y con mejores condiciones físicas y logísticas para llevar a cabo procesos productivos, aspectos que de alguna manera ya visualizaban en el acápite anterior. Sin embargo, estas manifestaciones de adaptación al cambio climático son inteligentes y en defensa de la vida propia y familiar, pero en muchos casos la premura generada por la emergencia no permite la planificación individual ni mucho menos colectiva.

Algunos ejemplos que soportan este tipo de apreciaciones son mi único respaldo para que mis opiniones no tengan una carga tan subjetiva. Muchos de los damnificados por el desbordamiento del río Munguidó o por la creciente del río San Juan, se encuentran hoy en Quibdó e Istmina respectivamente, porque familiares y amigos les brindaron y aun les brindan las condiciones para estar allí, dedicados a la venta informal de productos agrícolas o en el mejor de los casos como empleados de supermercados y lavaderos. En ambos casos la situación de emergencia los condujo a hacer lo primero que pudieron, pero no lo que estimaron podía ser una actividad que les brindara similares posibilidades a las que tenían; estas personas, según sus propias declaraciones anhelan tener condiciones materiales para regresar a su tierra y dedicarse a lo que siempre han hecho, que es casi lo único que saben hacer.

Los pescadores de Bahía Solano y Pizarro que fueron dotados de lanchas rápidas y mejores artes de pesca para evitar la fatiga que podrían traer consigo los largos viajes y las largas jornadas de trabajo, hoy encuentran en el mantenimiento de las embarcaciones una barrera para lograr los propósitos de quienes con muy buena intención les brindaron su ayuda. De tener estos esquemas de atención mejores procesos de planificación se tomarían todas las medidas para que resultaran realmente efectivas. Los agricultores del Medio Atrato, especialistas naturales en la cría libre de gallina criolla, han sido capacitados para el levante de pollo en cautiverio, los mismo ocurre con los pescadores continentales y los estanques piscícolas, la producción alcanza una o máxime dos cosechas, las razones del fracaso están casi siempre asociadas con aspectos administrativos, lo que permite concluir que se trata de procesos que no tienen una visión integral del asunto.

Con esta suerte de ejemplos y casos, la conclusión parece que no pudiese ser distinta; en las comunidades étnicas del Chocó Biogeográfico, los procesos de adaptación al cambio climático parecen ser reactivos, privados y autónomos. Sin embargo, la cada vez mayor toma de conciencia individual y colectiva podrían conducirnos a distintos y más efectivos caminos. El papel de grandes organizaciones étnicas como RECOMPAS, ASOCOETNAR, ASOMANO NEGRAS, ASOCONSEJOS, PCN, FECOVA, COCOMACIA, ASOCASAN, ACADESAN, ACABA, RISCALES, ASAIBA, OIA, UNIPA, ASO OREWA, ONIC, etc. va a resultar en extremo importante para estos propósitos. Estas organizaciones han mostrado un trabajo ordenado y persistente en procura de garantizar mejores condiciones de vida de la población; han mostrado interés comunitario y han liderado procesos de gran importancia para el futuro de la población, así como han operado proyectos y recursos importantes de la cooperación internacional, lo que no nos deja dudar de la validez de probarlos en las tareas de información y capacitación comunitaria, una vez se encuentren adecuadamente fortalecidas.

En casos aislados se observan procesos en los que se visualiza un mayor grado de planificación pública para lograr la adaptación de comunidades étnicas al cambio climático, pero finalmente las acciones suelen ser reactivas, tal es el ejemplo de la concertación de muy diversas entidades y comunidad para atender la situación de Olaya Herrera que vivió situaciones dramáticas por la apertura ya hace muchos años del Canal Naranjo. Algunas políticas estatales acentúan las dificultades al interior de las comunidades, la autorización de permisos de aprovechamiento minero y la fumigación de grandes áreas del territorio en procura de combatir los cultivos de uso ilícito son claras evidencias, con ellas no sólo se degrada masivamente el 


\section{Bioetnia Volumen 11, 2014}

territorio, sino que también se promueve el desalojo, el desarraigo, la persecución y la muerte; de igual manera, se destruye el hábitat de especies generando la desaparición de importantes elementos de la biodiversidad, al igual que se atenta contra la soberanía alimentaria y la salud de cientos de negros, indígenas y campesinos que están por fuera de este problema.

Tal vez la excepción a las medidas reactivas la constituye la participación de las comunidades empujando iniciativas de declaratoria de áreas dentro del Sistema Nacional de Áreas Protegidas con el ánimo de proteger ecosistemas y especies, y su trabajo en la intención de reglamentación de la Ley 70 en lo que tiene que ver con la constitución de una red de áreas protegidas en territorios colectivos de comunidades étnicas. Este párrafo es un reconocimiento a las organizaciones del Valle del Cauca que jugaron un papel preponderante en la declaratoria de Bahía Málaga como área protegida. Los efectos de esta decisión los indicará la historia, pero no se puede dejar de pensar lo que hubiese sido de un área de altísimo interés ecológico, utilizada en los fines que tenía pensado usarlo los empresarios del departamento del Valle.

\section{El papel de las organizaciones étnicas, de los entes territoriales y de la investigación en la implementación acciones planificadas de adaptación al cambio climático}

Los grupos étnicos del Chocó Biogeográfico poseen mecanismos autónomos de gobierno, de relacionamiento, de uso del territorio y de organización comunitaria, aspectos que llevan varios años en procesos de construcción, situación que me conduce a considerar el fortalecimiento de las organizaciones étnico territoriales, el fortalecimiento de los procesos propios de planificación de uso del territorio, el montaje de procesos de monitoreo continuo y el desarrollo de investigaciones pertinentes y concertadas, como los escenarios sobre los cuales se debe soportar la posibilidad de implementar acciones planificadas dirigidas a la adaptación de las comunidades de la región al cambio climático.

El camino recorrido por los procesos organizativos de comunidades negras e indígenas es largo y ha dado buenos frutos; las personas de manera individual creen y se deben a sus consejos y cabildos locales y estos a su vez hacen lo mismo con los mayores, por lo que considero que todas las acciones planificadas para abordar procesos de adaptación a cambio climático no sólo deben aprovechar este esquema organizativo, sino respetar lo que se considera en estricto el esquema de relacionamiento comunitario con cualquier entidad. Los consejos y cabildos locales y mayores, así como las asociaciones que han mostrado un fuerte trabajo en procura del bienestar colectivo de comunidades negras e indígenas son un referente obligado para estos fines, razón por la que su fortalecimiento se considera una necesidad para enfrentar estos retos.

El fortalecimiento de las organizaciones étnicas supone la necesidad de funcionamiento de sus espacios de discusión y gobierno autónomo, lo que obliga a avanzar en la perspectiva de realización cumplida de asambleas informativas y decisorias, que haga posible una participación amplia de un personal que se mantiene adecuadamente informado. El fortalecimiento de las organizaciones étnicas supone también la necesidad de garantizar la seguridad y la logística para una fluida movilidad entre los diferentes asentamientos humanos donde se encuentran los miembros de las organizaciones, además de la capacitación permanente en torno a temas técnicos y normativos de su interés para una mejor comprensión de los fenómenos que afectan sus territorios, lo cual sin duda se extiende a procesos de cambio climático, en el contexto de una educación propia que respete los modelos culturales al interior de las comunidades. Todos estos aspectos entre muchos otros que están contenidos en una estrategia de fortalecimiento organizativo, facilitarían la concertación de proyectos y acciones orientadas a la implementación de acciones preventivas, colectivas y planificadas, para la adaptación de las comunidades del Chocó Biogeográfico al cambio climático, las cuales a mi juicio son las ideales.

El siguiente paso consiste en establecer un vínculo entre las acciones que se planifiquen y los instrumentos de planificación tanto de los entes territoriales como de las organizaciones; me refiero por una parte a los planes y esquemas de ordenamiento territorial y por la otra a los procesos de zonificación y manejo ambiental del territorio, los planes de vida y los planes de etnodesarrollo con los que guían sus acciones las organizaciones étnico territoriales. En buena parte de los casos estos instrumentos de planificación de las organizaciones están construidos y los hemos estado revisando y consolidando en el marco del proyecto de zonificación y ordenamiento ambiental participativo de la reserva forestal del Pacífico y en los próximos años se debe extenders a toda la región. Sin embargo, en otra buena parte de casos, lo que se requiere es apoyar estos procesos de ordenamiento local y hacerlos coincidir con los presentados por los entes territoriales en los esquemas de ordenamiento territorial y por las corporaciones en cuanto a zonificaciones, planes de ordenación forestal, planes de manejo ambiental y planes de aprovechamiento y manejo forestal.

La implementación de acciones de adaptación no pueden darse sin un proceso de evaluación y seguimiento continuo, lo que supone adelantar estrategias de monitoreo capaces de medir si sus propósitos se están cumplimiento y tomar las medidas necesarias para garantizar que estas se dirijan 


\section{Cambio climático y percepciones culturales. W Klinger}

por el rumbo inicialmente trazado. El monitoreo requiere entonces que se involucren las entidades de la región según sus metas de competencia institucionales, los institutos de investigación y las universidades, las autoridades ambientales, los organismos de socorro, los entes territoriales, las autoridades de salud y eclesiásticas, las organizaciones no gubernamentales, cada una con competencias complementarias para provocar cambios sustanciales en la medida que su integración sea verdadera y efectiva. Se entiende desde este tópico que los procesos de capacitación orientados a elevar el nivel de conciencia ciudadana sobre el tema, no se pueden considerar efectivos sino generan cambios reales de actitud en la gente.

Por último, hago referencia al papel preponderante de la investigación concertada, más allá de producir documentos y cartografía con contenidos de zonificación y ordenamiento ambiental, se debe procurar que estos procesos se implementen y sean sostenidos en el tiempo, lo cual implica el desarrollo de investigación dirigida a producir información y conocimiento que haga esto posible; desde esta perspectiva se hace necesario conocer el estado y dinámica de los ecosistemas y de los procesos culturales dentro de las comunidades, las opciones de producción enmarcadas en modelos tradicionales de sostenibilidad ambiental, la apertura de nuevas opciones de producción que al tiempo que conservan los recursos naturales promueven el bienestar colectivo y los procesos de recuperación de áreas, ecosistemas y especies que puedan representar la generación de recursos económicos por la vía de la preservación de áreas que se constituyen de interés ecológico para la nación y el mundo, identificación de riesgos y amenazas.

De la mano de todo lo anterior, las comunidades encuentran urgente la vinculación de los entes territoriales y la autoridad ambiental para garantizar el rescate, el mejoramiento continuo y el impulso a los sistemas productivos ancestrales como una manera de contener los procesos productivos degradantes del medio natural, la gestión integral, reciclaje y transformación de residuos, la reducción de contaminantes, la producción de energías limpias, la reconversión hacia nuevos modelos de transporte, la sensibilización y educación ambiental, el ordenamiento ambiental del territorio, la recuperación de áreas degradadas y el fortalecimiento comunitario para la gobernanza territorial.

Hacer un frente comunitario e institucional común para defender el territorio de los embates de la naturaleza, pero sobre todo de quienes se aferran al aprovechamiento indiscriminado de los recursos que en él existen, promoviendo continuos hechos que violentan la seguridad de la región y la vida de líderes indígenas y negros, no sólo es una necesidad, sino también una obligación moral; hechos como los de Curvaradó y Jiguamiandó en el Chocó, Suárez en el Cauca, Saragoza en el Valle, Barbacoas en Nariño, son una vergüenza nacional que amerita la mayor atención de los organismos de control del Estado y una avanzada en busca de la solidaridad internacional para que los intereses y derechos colectivos de las comunidades negras e indígenas, no se vean atropellados por quienes sólo les interesa la acumulación de riqueza independientemente de los desastres ambientales y la vulneración de las costumbres y prácticas culturales que han soportado la existencia de los recursos con los que hoy cuenta el país, ese frente comunitario, social e institucional común, es sin duda la paz, por eso la apuesta sincera a escenarios sin guerra.

\section{La percepción comunitaria de algunos conceptos en relación con el cambio climático $y$ de sus derechos y deberes para afrontarlo}

En general la comunidad tiene una percepción adecuada de la mayoría de los conceptos básicos que se asocian con cambio climático y aunque no los define con precisión, conoce su significado. Los derechos y deberes de las comunidades para afrontar el cambio climático van mucho más allá de lo que se requiere para viabilizar la estrategia nacional de educación, formación y sensibilización de públicos frente al cambio climático, reconocido incluso por importantes líderes comunitarios de la región; el dinero fácil ha permeado algunas estructuras organizativas, por lo que se constituye en una obligación moral de consejos y cabildos, establecer mecanismos de control social que impiden la ocurrencia de hechos en los que las propias organizaciones posibilitan la implementación de métodos e intensidades de aprovechamiento no compatibles con las condiciones del territorio.

En conclusión, las comunidades entienden que su principal deber es defender el territorio de las prácticas productivas no concordantes con sus percepciones culturales, pero al mismo tiempo entienden que el ejercicio de su deber, requiere del reconocimiento de unos derechos sin los cuales no es posible cumplir con esas obligaciones morales; asuntos como el arraigo territorial y el respeto por las cosmovisiones y las diferencias culturales, son elementos obligatorios en las agendas de quienes por ley deben garantizar los derechos de las comunidades étnicas. En fin, los derechos de las comunidades se asocian con la garantía de condiciones fundamentales para permanecer con tranquilidad en el territorio, mientras que los deberes están asociados con hacer y permitir el uso de ese territorio en consonancia que sus preceptos culturales.

Volviendo a la estrategia nacional de educación, formación y sensibilización de públicos frente al cambio climático, la mayoría de mis entrevistados no la conocen, sin embargo, a mi juicio, conforme a sus respuestas, tienen absolutamente claros sus derechos y deberes para viabilizarla. Por una parte, 


\section{Bioetnia Volumen 11, 2014}

reclaman el derecho a estar adecuadamente informados y capacitados desde los niños hasta los ancianos, de expresar sus opiniones y de participar en todos los procesos que la implementación implique, y por otra parte, sienten que sus obligaciones giran en torno a actuar con conciencia sobre el tema, garantizando que sus acciones no generan mayor impacto negativo, para lo cual "se debe tener un cambio de actitud por parte de aquellos que no respetan las reglas de la naturaleza".

Como mecanismo para enfrentar el cambio climático, las comunidades aducen estar actuando en el marco de sus deberes; por una parte tienen en funcionamiento espacios de diálogos y comunicaciones internas a través del FISCH, donde confluyen comunidades negras e indígenas del Chocó para conocer y analizar información, así como para tomar decisiones sobre situaciones que afecten su territorio. De esta misma manera se convocan espacios regionales para mantener informadas a las comunidades sobre situaciones adversas a sus lógicas organizativas, los cuales cuentan con representación de FISCH y donde se comunican las decisiones tomadas en el departamento del Chocó. Desde esta misma perspectiva las comunidades negras e indígenas trabajan intensamente en varios proyectos locales $\mathrm{y}$ en el marco de estos proyectos revisan y ajustan las propuestas de ordenamiento ambiental de sus territorios, como mecanismo para no generar conflictos de uso que repercutan negativamente en los fenómenos relacionados con el cambio climático.

Los grandes problemas de la región que tienen relación con el cambio climático siguen estando, según las comunidades, en las autoridades que siguen permitiendo el aprovechamiento de recursos dentro de territorios colectivos, incluso con órdenes judiciales de desalojo de las comunidades allí presentes, el aprovechamiento ilegal en pesca, minería y forestería, el cual en un buen número de casos cuenta con brazos armados que intimidan y desplazan a la gente del territorio, y el crecimiento del narcotráfico que avanza por la fuerza y sin control de la ley. Estas actividades por su intensidad o por sus procedimientos tiene impactos sobre el cambio climático o sobre sus efectos, lo que se evidencia en los resultados que hoy se tienen en la región. Las comunidades así lo perciben, así que a mi juicio se prevé un camino expedito para lograr una información pública nunca antes vista en materia ambiental.

\section{A manera de conclusión}

Las comunidades negras e indígenas del Chocó Biogeográfico son cada vez más conscientes del cambio climático y de sus efectos, los perciben de diversas maneras conforme al grado de conocimiento tradicional y académico que tengan y en relación con la cosmovisión y su relación histórica con la naturaleza. Desastres naturales, escasez de alimentos y recursos naturales en general, aparición de plagas, insalubridad y epidemias, variación en la temperatura y el régimen de lluvias, entre otros aspectos, son vistos por la población en relación con el cambio climático.

De igual manera, manejan los conceptos básicos que se asocian con el cambio climático y son conocedores de sus deberes y sus derechos frente al tema, consideran estar actuando para enfrentarlo, pero piensan que si logran mayores niveles de capacitación y de fortalecimiento de sus organizaciones obtendrían mejores resultados. Destacan el papel de las instituciones como complementarios en el propósito de enfrentar el cambio climático y reconocen que no adelantan acciones planificadas y preventivas para sobrellevar el fenómeno.

La investigación juega un papel preponderante en la consolidación y recuperación de los elementos culturales que han mostrado históricamente una adecuada relación hombre-naturaleza, en la identificación de especies, ecosistemas y procesos productivos de limpios, en el ordenamiento general del territorio, pero también visualizan el ejercicio de la autoridad ambiental como preponderante para el logro de buenos resultados, reducir la contaminación y adelantar proyectos de fomento que brinden alternativas de generación de ingresos para no perturbar notoriamente los ecosistemas, son algunas tareas de las que se les asigna. 